\title{
COVERT ATTENTION LEADS TO FAST AND ACCURATE DECISION MAKING
}

(C) 2022, AMERICAN PSYCHOLOGICAL ASSOCIATION. THIS PAPER IS NOT THE COPY OF RECORD AND MAY NOT EXACTLY REPLICATE THE FINAL, AUTHORITATIVE VERSION OF THE ARTICLE. PLEASE DO NOT COPY OR CITE WITHOUT AUTHORS' PERMISSION. THE FINAL ARTICLE WILL BE AVAILABLE, UPON PUBLICATION, VIA ITS DOI: 10.1037/XAP0000425

\author{
CSonja Perkovic*\# \\ Department of Management / MAPP \\ Aarhus University \\ 8210 Aarhus V, Denmark \\ sp [at]mgmt . au .dk \\ (1)Carl-Johan Lagerkvist \\ Department of Economics \\ Swedish University of Agricultural Sciences \\ 75007 Uppsala, Sweden \\ carl-johan. lagerkvist [at]slu.se
}

\author{
D Martin Schoemann \# \\ Department of Management \\ Aarhus University \\ 8210 Aarhus V, Denmark \\ $\mathrm{ms}$ [at] mgmt.au.dk
}

(1) Jacob L. Orquin

Department of Management / MAPP

Aarhus University

8210 Aarhus V, Denmark

jalo[at]mgmt.au.dk

January 27,2022

\begin{abstract}
Decision makers are regularly faced with more choice information than they can directly gaze at in a limited amount of time. Many theories assume that because decision makers attend to information sequentially and overtly that is, with direct gaze, they must respond to information overload by trading off between speed and decision accuracy. By re-analyzing five published studies, we show that participants, besides using overt attention, also use covert attention. That is, without being instructed to do so participants attend to information without direct gaze to evaluate choice attributes that lead them to either choose the best or reject the worst option. We show that the use of covert attention is common for most participants and more so when information is easily identifiable in the peripheral visual field due to being large or visually salient. Covert attention is associated with faster decision times suggesting that participants might process multiple pieces of information simultaneously using distributed attention. Our findings highlight the importance of covert attention in decision making and show how decision makers may be gaining speed while retaining high levels of decision accuracy. We discuss how harnessing covert attention can benefit consumer decision making of healthy and sustainable products.
\end{abstract}

Keywords covert attention $\cdot$ peripheral vision $\cdot$ decision making $\cdot$ consumer choice $\cdot$ eye tracking

\footnotetext{
${ }^{*}$ Corresponding author;

The authors thank Hrvoje Stojic and Michael Schulte-Mecklenbeck for helpful comments and Helena Juliet Kahan for editing. This research was supported by the Independent Research Fund Denmark (grant number 8046-00014A) and Lundbeckfonden (grant number R281-2018-27). The funders had no role in conceptualization, data analysis, decision to publish, or preparation of the manuscript; the authors declare no competing interests. All datasets and analysis scripts will be openly available at: https://osf.io/nfudq. "Sonja Perkovic and Martin Schoemann contributed equally to this article and the authors are listed in alphabetical order.
} 


\section{Introduction}

Decision makers are regularly faced with more choice information than they can directly gaze at in a limited amount of time. Even a small supermarket can feature over 100 products per category (Perkovic \& Orquin, 2018) making it impossible to process everything and relevant information can, therefore, fail to attract our attention. Nutrition, health, or sustainability information on products is, for instance, often not gazed at by consumers (Bartels et al., 2018; Orquin et al., 2020). Rather than blaming consumers for lack of motivation, it is necessary to understand that more than half of the variance in decision makers' attention is determined by visual factors such as the surface size, visual salience, and position of information (Orquin et al., 2021). These visual factors can help decision makers to attend to information relevant to their own goals, but visual factors are ultimately controlled by producers and retailers (Orquin \& Wedel, 2020). Retailers, for instance, rely heavily on visual factors to guide consumer attention with large and colorful sales promotions. In product design, producers generally prioritize brands and logos by making them consistently larger, more salient, and more centrally positioned than nutrition or sustainability information. This general structure in the visual environment leads consumers to attend to brands and logos at the expense of attention to nutrition and sustainability information (Orquin et al., 2020). The design of product packaging information, thus, seems to impede attention to information that would enhance the ability to choose healthy and sustainable products. On the other hand, product design principles like associating shape and color with some product information (e.g., brand) could benefit decision makers. Shape and color are guiding cues that can be perceived across a wide visual field (Wolfe \& Horowitz, 2017). When coupled with meaningful product dimensions, these cues allow decision makers to screen out multiple irrelevant products in parallel by focusing on those products that have a desired color or shape (e.g., red with curved sides or green with straight sides). Consumers can, for instance, covertly identify relevant product category using their peripheral visual field before attending to them overtly (Wästlund et al., 2018), that is, with direct gaze, thus saving time and reducing the burden of visual search. Could similar principles benefit decision makers when evaluating individual products based on product information? In other words, is it strictly necessary to attend directly to each piece of product information or could multiple pieces of information be processed in parallel? And if so, what would that require in terms of visual product design? Previous research on decision makers' attention has, for the most part, taken the view that we process information sequentially by directly attending to each piece of information (Stüttgen et al., 2012; Thomas et al., 2021). ${ }^{2}$ In the following section, we review theory on attention and decision making and compare this to findings from vision science that suggest that the assumption of sequential and direct visual search may need to be reexamined. We then meta-analyze four published data sets and show that participants in these studies rely on both overt and covert attention when making choices. Next, we demonstrate the same effects in a separate published data set from a more naturalistic food-choice task. Finally, we discuss our findings in relation to decision theory and provide recommendations for how to improve consumer decision making by harnessing the potential of covert attention.

\subsection{Attention and choice}

A popular class of models that explain decisions under time constraints are based on sequential sampling and accumulation to threshold principles (Busemeyer et al., 2019). These models assume that the decision maker accumulates evidence for each available choice option until a decision-threshold is reached, at which time a decision is made in favor of the option that reaches the accumulation threshold. These models have been remarkably successful in explaining both choices and response times in perceptual, memory, and categorization tasks (Forstmann et al., 2016; Ratcliff et al., 2016), which have objectively correct answers and produce very consistent decision-making behavior. However, preferential choices do not have objectively correct answers and produce rather inconsistent decision-making behavior which depends on the subjective goals and preferences of the individual decision maker (Busemeyer et al., 2019; Dutilh \& Rieskamp, 2016). To better understand preferential decision-making behavior, it has become evident that models benefit from incorporating attention in addition to the sequential sampling principle (Turner et al., 2018). For a long time, attention was treated as a latent, stochastic variable in the sequential sampling process (Busemeyer et al., 2019; Turner et al., 2018) $)^{3}$, but direct measures of visual attention have recently been used to constrain the sequential sampling models of preferential choice. Krajbich et al. (2010), for instance, extended the drift diffusion model (Ratcliff, 1978) to account for the role of visual attention proposing that evidence for a choice option is sampled while the option is overtly attended to (for a review, see Krajbich, 2019). Put differently, gazing at an option accumulates evidence in favor of the option and increases the probability of it being chosen (Gluth et al., 2020; Smith \& Krajbich, 2018, 2019; Thomas et al., 2021; Thomas et al., 2019). These models posit a relation between direct gaze and a neural value signal (Hare et al., 2011; McGinty et al., 2016) so that the value signal increases for the gazed-at object and simultaneously

\footnotetext{
${ }^{2}$ As an exception to this general trend, the iCodes model assumes parallel cognitive processing of information (Jekel et al., 2018).

${ }^{3}$ For most multi-alternative, multi-attribute preferential choice models, the allocation of attention follows a Bernoulli process where each attribute is stochastically and hence sequentially attended to with probabilities proportional to their respective attention weights in the model (Turner et al., 2018, p. 336).
} 
diminishes for all not gazed-at objects. Other models have been proposed with slightly different assumptions (Ashby et al., 2016; J. F. Cavanagh et al., 2014; Colas, 2017; Fisher, 2017; Gluth et al., 2018; Towal et al., 2013). In general, all sequential sampling models incorporating visual attention provide a good fit to observed choices, eye movements, and response times and have furthermore been corroborated by a large body of studies pointing to an effect of direct gaze on choices (Armel et al., 2008; Fisher, 2021; Ghaffari \& Fiedler, 2018; Kunar et al., 2017; Lim et al., 2011; Liu et al., 2020; Milosavljevic et al., 2012; Pärnamets et al., 2015; Shimojo et al., 2003; Tavares et al., 2017).

While there is much evidence to support the notion of decision making as a sequential sampling process, some recent findings have cast doubt on the assumption. Many sequential sampling models assume, and empirically demonstrate, that because choice options are evaluated sequentially, the location of the first fixation is independent of option values (J. F. Cavanagh et al., 2014; Krajbich \& Rangel, 2011). However, it has recently been demonstrated that monkeys trained to make preferential choices between two familiar options gaze at the option with the highest value first, suggesting that they covertly (i.e., without gazing directly) assess the value of both options and direct their gaze to the one with higher value (S. E. Cavanagh et al., 2019). In this study, covert attention guided gazes and mediated choices, which were better explained assuming the use of covert attention and not just evidence accumulation based on overt attention. The finding dovetails with research on human subjects showing that participants can covertly process relevant information and use it for deploying overt attention (Towal et al., 2013), such as evaluating eight options and directing a fixation within 0.50 seconds to the option that best combines reward value and visual salience according to an ideal Bayesian observer (Navalpakkam et al., 2010). It has also been shown that participants use covert attention to guide overt attention towards relevant choice options both in lab and field studies conducted in visually complex retail environments (Wästlund et al., 2018). These studies suggest that covert attention may play a role in directing overt attention in decision making and therefore question the assumption that evidence in favor of or against choice options is exclusively sampled through sequential overt attention.

While covert attention remains relatively under-researched in the context of decision making, it is well documented in many areas of vision science (Carrasco, 2011). The feature integration theory of attention was based on the finding that visual search for a single target can be performed in parallel or sequentially. The feature integration theory proposes two stages of visual search. First, visual features are processed in parallel. If the initial parallel processing is not sufficient to discriminate the target, the observer then performs a slower serial search process (Treisman \& Gelade, 1980). Parallel search is sufficient in tasks where the target is defined by a single feature, such as a red target among green distractors, and can thus be found using distributed attention. Additional sequential search occurs in tasks with a conjunction of features, such as a red horizontal line among red and green vertical and horizontal lines, where the target cannot be identified using distributed attention. Hence, in situations in which the target can be identified using only information from the initial, parallel stage, parallel search outperforms sequential search regarding the time it takes to find the target (Treisman \& Gelade, 1980). Later, the guided search model demonstrated that even search for conjunction targets can involve some information from the initial, parallel stage, making some conjunction searches faster than previously theorized (Wolfe, 1994; Wolfe et al., 1989). Distributed attention is also well known in research on scene viewing where it has been shown that observers can extract the gist of a visual scene through covert attention and, within the duration of a single fixation, recognize the scene and accurately classify a few objects (Greene \& Oliva, 2009; Oliva, 2005). Medical doctors can, for instance, classify radiology images based on scene gist and make above chance diagnoses in as little as 0.25 seconds (Evans et al., 2013). This ability, however, may require extensive experience and even so the level of accuracy seems to be modest. There is also evidence suggesting that covert attention can be deployed to a more focused spatial location. Techniques such as Posner cueing (Posner, 1980) can, for instance, facilitate or suppress covert attention in a specific location. Focused covert attention is also evident in real-world tasks such as in social interactions (Dosso et al., 2020) and sports (Hausegger et al., 2019) where, for instance, martial arts experts anchor their gaze on the opponent's body in locations that allow optimal covert detection of attacks. Covert attention in sports seems to, however, depend on experience (Kato, 2020).

Considering the findings from vision science, it is perhaps less surprising to learn that covert attention can guide overt attention in decision making (S. E. Cavanagh et al., 2019; Navalpakkam et al., 2010; Towal et al., 2013; Wästlund et al., 2018). Rather, the question then is whether covert attention plays a role in preferential choice beyond that of being a precursor to overt attention? Following the insights from vision science, covert attention should in principle allow decision makers to identify and evaluate multiple objects simultaneously without the need for subsequent overt attention. Without a need for subsequent overt attention, covert attention might provide decision makers with benefits in terms of speed or accuracy which is highly relevant to many every-day decisions. There is indeed some evidence that decision makers can make fast and accurate choices based on covert attention without any subsequent overt attention when they are instructed to do so in an experiment (Milosavljevic et al., 2010).

It seems then, that decision makers can use covert attention as a precursor to overt attention under some conditions, but do decision makers voluntarily use covert attention in every-day decisions i.e., when not explicitly instructed to do so? Whether decision makers use covert attention may depend on certain conditions. For example, the participants in 
S. E. Cavanagh et al. (2019) received extensive training on a small and visually distinct set of stimuli, and the pairs of stimuli between which the subjects chose varied along a single value dimension. Since the values were overlearned and the information load was fairly low, it may not have been necessary to fixate the stimuli to make an optimal choice. In Towal et al. (2013), all choice options were familiar to participants and were presented in a $2 \times 2$ visual array, which may have facilitated covert recognition of the options. In the experiments by Navalpakkam et al. (2010), stimuli contained only two dimensions (i.e., shape and orientation or shape and brightness), and varied along only one of these dimensions. In addition, the two target options always represented the endpoints of the stimulus dimension that was varied on a given trial and all distractors had the same stimulus value. This likely made it easier for participants to covertly locate the two target objects. In the in-store study by Wästlund et al. (2018), participants mainly used covert attention when target and distractor products were visually distinct. When target and distractors were visually similar, participants did not rely on covert attention. This suggests that the use of covert attention may depend on whether stimuli are visually distinctive and perhaps on familiarity with the stimuli and task.

We address these questions in two studies. In Study 1, we extract and synthesize data from four published articles on the likelihood of choosing the dominant (best) option, conditional on having gazed at the necessary attribute to identify the option as dominant. We show that decision makers accurately choose the dominant option above chance even without gazing at the necessary attribute. This suggests that information from covert attention is directly integrated in the decision process without a need for subsequent overt attention. The use of covert attention increases when stimuli are visually distinct, but covert attention does not seem to depend on a high degree of learning. We also show that trials with accurate choices and no direct gaze to the necessary attribute are on average faster than those with direct gaze, which corroborates the theoretical benefit of covert distributed attention on speed. In Study 2, we demonstrate the generalizability of our findings from Study 1 by re-analyzing data from another published article.

\section{Study 1}

In Study 1, we re-analyzed four published data sets (Orquin et al., 2020; Orquin et al., 2013; Orquin \& Lagerkvist, 2015; Peschel et al., 2019) with a total sample of 393 participants. The data sets share several features which make them suitable for quantifying the role of covert attention in decision making. In all data sets participants made a series of one-shot choices in choice sets of two to four multi-attribute options. One of the choice options is always considered the target option which means that it dominates the non-target option on account of a single target attribute (Figure 1). The appearance of the target attribute was manipulated across trials - termed discriminability - and the manipulations differed slightly between data sets. For details about the data sets, please see Appendix A.

\subsection{Method}

\subsubsection{Extraction and synthesis of effect sizes}

To assess the magnitude of overt vs covert attention, we computed the relative effect of direct gaze on choice in favor of the target option as an Odds Ratio (OR). We computed the OR as (S3/S4)/(S1/S2), where S1 and S2 are trials in which the target attribute is not fixated on and the target option is chosen or not, respectively, whereas S3 and S4 are trials in which the target attribute is fixated on and the target option is chosen or not, respectively (Figure 1e). The OR thus reveals the effect of direct gaze in terms of its ratio to the effect of no direct gaze. To obtain $95 \%$ confidence intervals (CI) and the standard errors (SE) of ORs, we generated 10,000 bootstrap samples for each data set. We then extracted effect sizes separately for the trials that differed in the discriminability of the target label, that is, in size (small vs large) or in salience (low vs high). To synthesize the effect sizes, we transformed each OR and corresponding SE to $\log$ scale and then performed a random effects inverse variance meta-analysis (Borenstein et al., 2011). We extracted response time (RT) effect sizes as the RT difference between trials where the target option was chosen but the target attribute was either fixated on or not (Figure 1e, S3 and S1). RT effect sizes were then synthesized using a random effects inverse variance meta-analysis.

\subsubsection{Data processing}

We used R (R Core Team, 2020) for data processing. To perform our analysis independently of previous individual analyses' choices, such as area-of-interest (AOI) definitions or data exclusions, we used raw eye-tracking data ${ }^{4}$. This was particularly important for the AOI definitions, as our analysis crucially depends on the choice of AOI margins when determining whether the target attribute had been fixated on or not. To account for this dependency, we computed S1, S2, S3, and S4 (Figure 1) as well as all derived statistics (i.e., ORs and SEs) using a theoretically plausible and

\footnotetext{
${ }^{4}$ We used the eye-tracking data as delivered by the respective host system, that is, fixations and saccades classified but not connected to stimuli material.
} 
Figure 1: Example stimuli from data sets included in Study 1

a

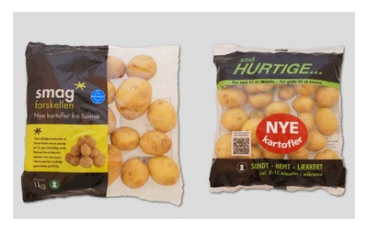

e

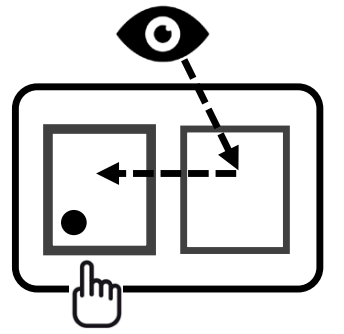

S1

target option chosen target attribute not fixated

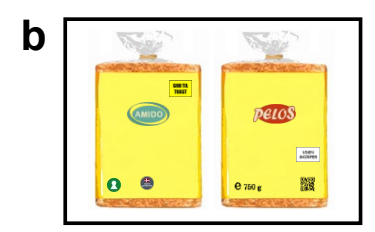

C

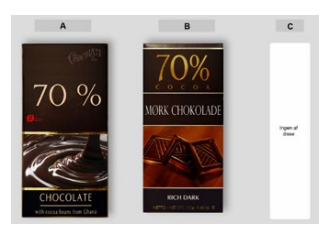

d

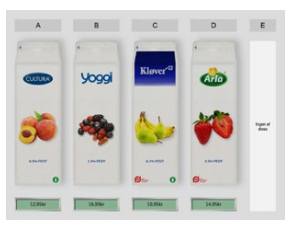

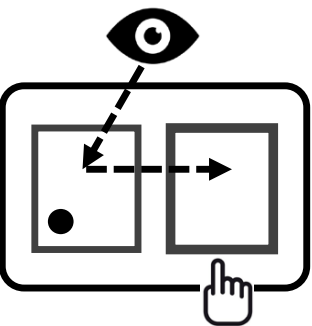

S2

target option not chosen target attribute not fixated

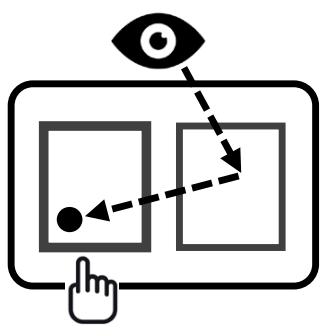

S3

target option chosen target attribute fixated

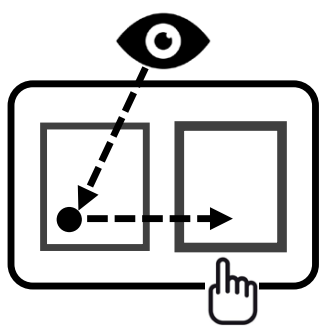

S4

target option not chosen target attribute fixated

(a) Orquin and Lagerkvist (2015) (b) Orquin et al. (2020) (c) Peschel et al. (2019) (d) Orquin et al. (2013) (e) Participants chose between options that mainly differed on account of a randomly positioned target attribute. The figure illustrates a choice set with two options and the target attribute on the left option (black circle). The option with the target attribute is the target option. Participants could either (S1) not fixate on the target attribute and choose the target option, (S2) not fixate on the target attribute and not choose the target option, (S3) fixate on the target attribute and choose the target option, or (S4) fixate on the target attribute and not choose the target option. For a colored figure, please see the online version.

justifiable AOI margin in each data set first, followed by a sensitivity analysis over a range of margins that are feasible for the included data sets and commonly used.

In two data sets (Orquin et al., 2020; Orquin et al., 2013), the stimuli were semi-naturalistic or non-naturalistic which is often characterized by relatively large distances between AOIs. In such cases, it has been proposed to determine the optimal AOI margin based on the capture rate which depends on the stimulus size and shape as well as the accuracy and precision of the respective eye tracker (Orquin \& Holmqvist, 2018). We followed this proposal and performed a grid search for the optimal AOI margin based on maximizing the capture rate. In the other two data sets (Orquin \& Lagerkvist, 2015; Peschel et al., 2019), the stimuli were naturalistic which is, contrary to the non-naturalistic stimuli, often characterized by a relatively narrow layout. In such cases, the optimal AOI margin should minimize the rates of false negatives and false positives (Orquin et al., 2016).

\subsection{Results}

We based our analyses on the following assumption. If participants were unaware of the target attribute, the probability of choosing either of the options should approach chance level. Alternatively, they should be more likely to choose the target option. We could then examine whether directly gazing at the target attribute increases the likelihood of choosing the target option, as commonly assumed, by inspecting fixation patterns and choice frequencies. If participants relied on overt attention for processing relevant stimuli, we should observe a large proportion of choices in favor of the target option when the target attribute is fixated on. If participants also relied on covert attention, we should observe an above chance level proportion of choices in favor of the target option when the target attribute is not fixated on. Hence, by calculating the choice likelihood for the target option depending on whether the target attribute was fixated on or not, we obtained a ratio indicating the relative use of overt vs covert attention. 
Figure 2: Main analysis: Decision makers use both covert and overt attention to make choices

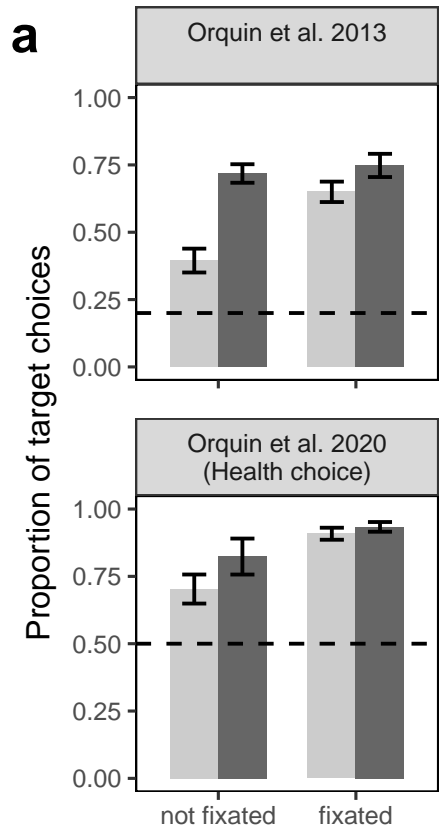

b
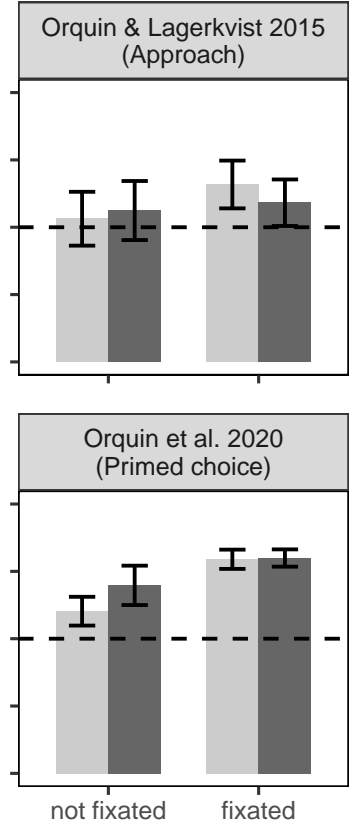

Target attribute fixation
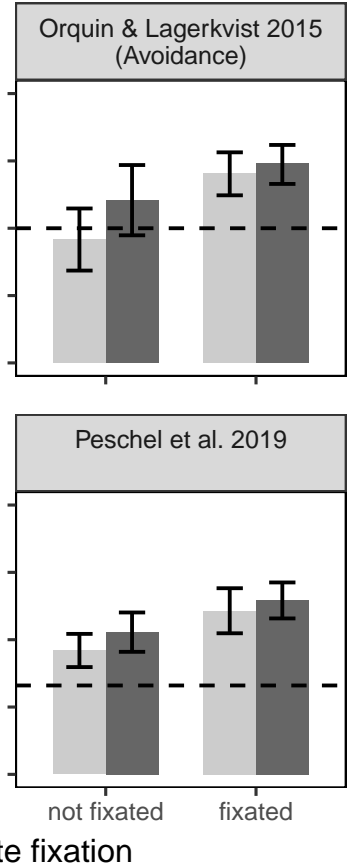

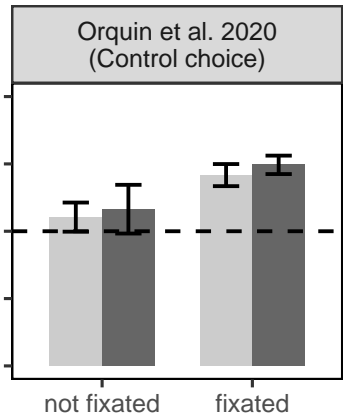

Discriminability

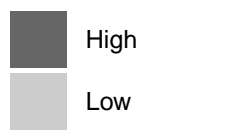

Discriminability $\rightarrow$ High $\rightarrow$ Low

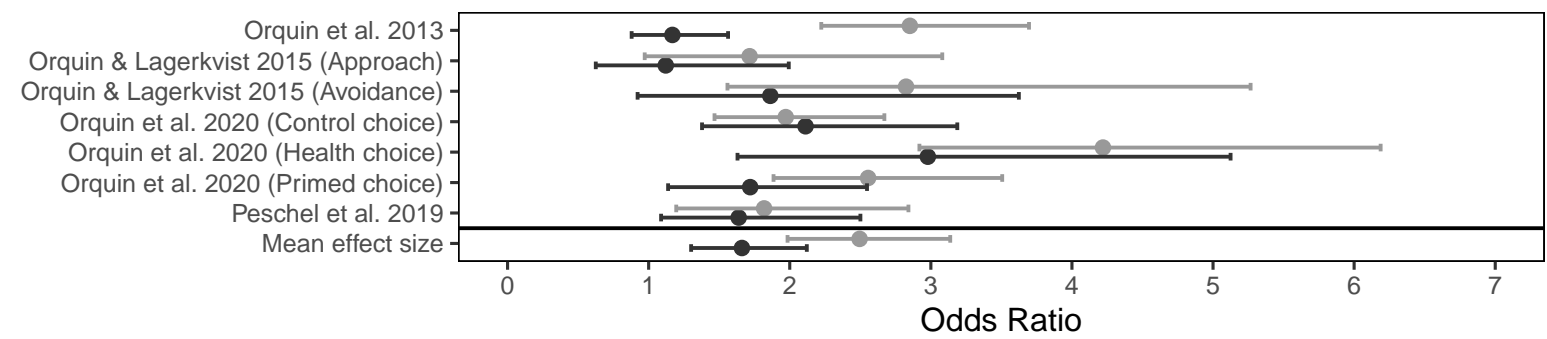

c

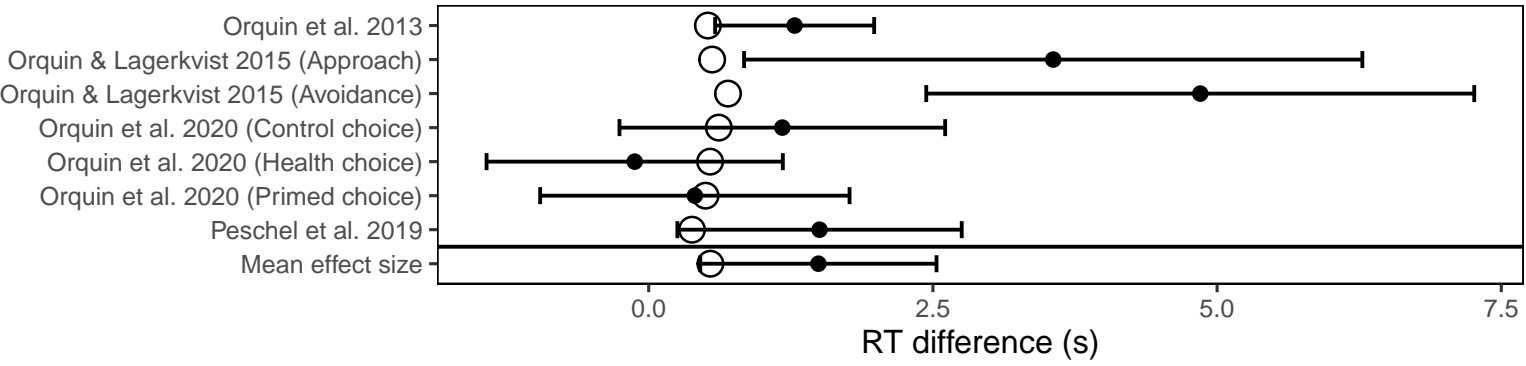

(a) Probability of choosing the target option depending on fixations to target attribute and discriminability condition. Error bars represent bootstrapped 95\% confidence intervals and horizontal lines represent chance level choice. (b) Forest plot of the effect of covert vs overt attention on choice for each discriminability condition. Error bars represent bootstrapped $95 \%$ confidence intervals. (c) Forest plot of the effect of covert vs overt attention on response times (RT) as untransformed coefficients from linear mixed models. Error bars represent $95 \%$ confidence intervals. Empty circles indicate the average time participants spent overtly gazing at the target attribute when the target option was chosen (Figure 1e, S3). 


\subsubsection{Choice proportions}

We began by investigating participants' proportions of choosing the target option given discriminability (high vs low) and fixation to the target attribute (yes vs no). We found that participants were in general more likely to choose the target option if they had fixated on the target attribute (Figure 2a). As indicated by the confidence intervals, even when not fixating on the target attribute, participants still chose the target option above chance in four out of seven data sets independent of attribute discriminability. When not fixating on the target attribute, participants were always more likely to choose the target option in the high discriminability compared to the low discriminability condition although confidence intervals for high and low conditions overlapped for all except one data set.

\subsubsection{Effect of covert attention}

To quantify the role of covert attention, we calculated ORs $(O R=(S 3 / S 4) /(S 1 / S 2)$, Figure 1e) from pooled trials across participants for each data set and condition separately, with an $\mathrm{OR}=1$ indicating that participants were equally likely to choose the target option regardless of whether the target attribute was fixated on or not. A closer inspection shows that across all data sets, participants made on average $28.88 \%$ choices of the target option when the target attribute was not fixated on and that almost all participants contributed to this effect (Figure 5). Higher OR indicates that participants were more likely to choose the target option if the target attribute had been fixated on. As expected, the OR is higher for low than for high discriminability attributes in all except one condition (Figure 2b). This implies that fixating on the target attribute had a smaller effect on choice accuracy in the high discriminability condition compared to the low discriminability condition. The meta-analysis of the log-transformed ORs supports the observed pattern with a significant difference between high and low discriminability conditions, $Q(1)=5.65 ; p=0.017$, with a lower (back-transformed) $\mathrm{OR}$ in the high discriminability condition, $\mathrm{OR}=1.66 ; 95 \% \mathrm{CI}=[1.30,2.12] ; p<0.001$; $I^{2}=50.99$, than in the low discriminability condition, $\mathrm{OR}=2.50 ; 95 \% \mathrm{CI}=[1.98,3.14] ; p<0.001 ; I^{2}=61.32$. Both conditions have medium levels of heterogeneity as expressed in the $I^{2}$ values. Inspecting Figure $2 \mathrm{~b}$ suggests that some of the heterogeneity could stem from the Orquin et al. (2020, Health choice) data set. Omitting this observation reduced heterogeneity to $I^{2}=36.09$ and $I^{2}=29.59$ for the high and low discriminability conditions, respectively.

To get a better understanding of the decision process behind target choices when the target attribute is fixated on vs not fixated on, we next examined participants' response times (RTs) irrespective of discriminability. RTs have been associated with different aspects of the decision processes with longer RTs generally assumed to indicate more deliberate choices (Payne et al., 1988) and being associated with a higher level of accuracy (Forstmann et al., 2016). To analyze the effect of covert attention on participants' RTs, we fitted linear mixed models to the trials in which participants chose the target option as a function of target attribute fixation (binary: yes vs no) and trial order (continuous: $[0,+\infty]$, first trial set to 0 ). For this mixed-model analysis we omitted the discriminability condition because separate analyses for low and high discriminability would have resulted in too few data points in the data sets to obtain reliable model fits. The estimated coefficients for the RT difference due to covert attention (i.e., the fixed effect of target attribute fixation) and the corresponding standard errors were then extracted for a meta-analysis (for detailed results for each data set, see Table 3). For the sake of comparison, we also computed the mean fixation time on the target attribute for trials where the target option was chosen $M_{D T}=0.54$ seconds. The mean fixation time indicates the expected reduction in RT due to not having attended to the target attribute. The meta-analysis of RT difference showed a large effect of covert vs overt attention with covert trials being on average 1.50 seconds shorter, $\mathrm{RT}=1.49 ; 95 \% \mathrm{CI}=[0.45,2.53] ; p=0.005 ; I^{2}=74.49$ (Figure 2c). In other words, trials where the target attribute was fixated on took longer than trials where the target attribute was not fixated on. With trials lasting on average 7.04 $(S E=0.06)$ seconds, this corresponds to $21.21 \%$ reduction in the total RT (Figure 2c). See Table 4 for a detailed summary of descriptive statistics calculated from the four data sets.

\subsubsection{Learning effects}

To examine learning effects on the use of covert attention, we used the same analytical approach as in our analysis of participants' RTs. However, in this analysis we fitted binomial mixed models to participants' covert target choices (i.e., binary: S1 vs S2-S4, Figure 1e) as a function of trial order. Again, we omitted the discriminability condition in this mixed-model analysis because separate analyses for low and high discriminability would have resulted in too few data points in the data sets to obtain reliable model fits. The estimated coefficients for the prevalence of covert target choices at trial 1 (i.e., the intercept) and the probability difference of covert target choices due to learning over trials (i.e., the fixed effect of trial order) as well as the corresponding standard errors were then extracted for a meta-analysis (for detailed results for each data set, see Table 2). The meta-analysis showed a small increase in the probability of covert target choices for each performed trial, $\mathrm{OR}=1.01 ; 95 \% \mathrm{CI}=[1.01,1.01] ; p<0.001 ; I^{2}=4.46$. However, 
Figure 3: Sensitivity analysis: The effect is not an artefact of improper area of interest assignment
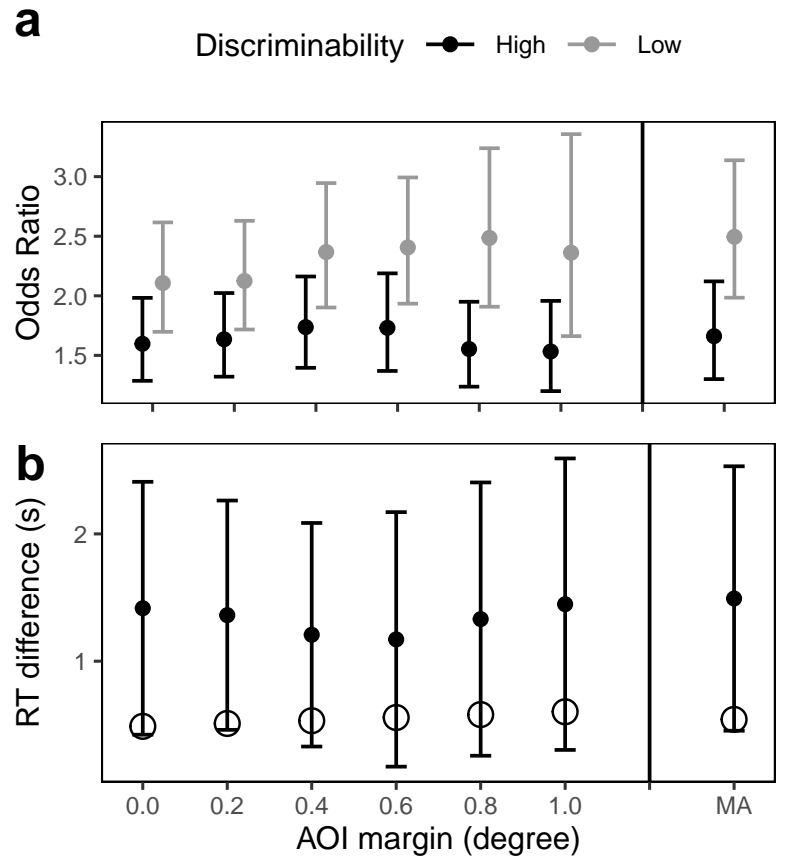

Overall, the results are stable across different areas of interest margins (x-axis). The margin used in the main analyses is labelled as MA. (a) Results for the effect of covert vs overt attention on choice. (b) Results for the effect of covert attention on response times.

the meta-analysis of the intercept showed that the prevalence of covert target choices is relatively high from the first trial, $\operatorname{Pr}=0.30 ; 95 \% \mathrm{CI}=[0.17,0.46] ; p=0.018 ; I^{2}=92.50$. Therefore, these results indicate that the use of covert attention increases marginally with, but is not dependent on, task experience.

With regard to RTs, we evaluated the remaining coefficients from our previous RT analysis (see the section above) in which we modeled the RT of target choices as a function of target attribute fixation and trial order. The estimated coefficients for the RT difference due to learning over trials (i.e., fixed effect of trial order) and its interaction with the RT difference due to covert attention (i.e., fixed interaction effect of target attribute fixation and trial order) as well as the corresponding standard errors were then extracted for a meta-analysis (for detailed results for each data set, see Table 3). The meta-analysis showed a small decrease in participants' RT for each performed trial, RT $=-0.03$; 95\% CI $=[-0.06,-0.01] ; p=0.013 ; I^{2}=58.17$, presumably due to learning effects (Orquin et al., 2013). However, the meta-analysis also showed that there is no significant interaction with covert attention, $\mathrm{RT}=0.00 ; 95 \% \mathrm{CI}=$ $[-0.02,0.01] ; p=0.781 ; I^{2}=0.03$. Therefore, these results indicate that the advantage of covert attention with regard to faster RT is stable over time and is not just a product of task experience.

\subsubsection{Sensitivity analysis}

To test the validity of our main results, that is the effect of covert attention on choice and its effect on RTs, we performed a sensitivity analysis (Carter et al., 2019). One of the possible reasons for accurate choices where the target attribute was not fixated on could be inappropriate AOI margins that fail to detect fixations to the target attribute. Specifically, if a fixation to the target attribute falls outside the AOI, it is not detected and thus the trial would be classified as a trial where the target option was chosen and the target attribute was not fixated on (i.e., S1, Figure 1e). To test the sensitivity of our results for the chosen AOI margin, we performed the main analyses over a range of feasible and commonly used AOI margins, $m=\left[0.00^{\circ}, 0.20^{\circ}, 0.40^{\circ}, 0.60^{\circ}, 0.80^{\circ}, 1.00^{\circ}\right]$ (for more details, see the Data processing section). The sensitivity analysis showed that the main results do not critically depend on a specific AOI margin for both the effect of covert vs overt attention on choice and the effect of covert attention on RTs (Figure 3). 


\subsection{Discussion}

In Study 1, we re-analyzed four published data sets to examine the use of covert attention in preferential decision making. Our results indicate that participants use covert attention to in- and exclude choice options based on relevant target attributes and that choices with covert attention are on average faster. The findings challenge models which assume that decision makers mainly or only accumulate evidence during direct fixations to choice options and attributes (e.g., Krajbich et al., 2010), but support models assuming parallel processing of information (Jekel et al., 2018). Whether the findings can be generalized to real-world behavior depends, of course, on the external validity of the included studies. It is worth noting that all included studies used choice sets that are smaller than what can be expected in typical consumer contexts which may have facilitated the use of covert attention.

\section{Study 2}

In Study 2, we re-analyzed another published data set to examine whether decision makers use covert attention in choice tasks that match real-world consumer contexts in terms of the number of choice options and the discriminability of target attributes. The new data set shares the same relevant features as the data sets in Study 1 but has a more externally valid study design that resembles an online grocery store (Perkovic \& Orquin, 2018). The hypotheses are derived from the findings of Study 1 and we expected to find similar effects (point estimates) 5 .

\subsection{Method}

The data set contains 78 participants who each made 50 choices. The stimuli were based on a real-world online grocery store (using the website layout of osuma.dk from 2015) and each choice set contained 8 unique products. Choice sets represented different food categories and the study resembled a large-basket shopping trip where the consumer purchases from multiple categories such as bread, dairy, meat, ready meals, and so forth (Martin et al., 2020). Participants were instructed to choose the healthiest product in each trial. Each product contained a small image of the product, the brand name, quantity, price, and index price (Figure 4a). Two additional labels - the Keyhole label and the Danish organic label-were randomly assigned to products in such a way that co-occurrence of the two labels varied across three conditions $(25 \%, 50 \%$, and $75 \%$ co-occurrence). There were four Keyhole labels and four organic labels in each choice set. The discriminability of the two labels was not manipulated, but differences arise naturally since some products are larger, which creates crowding around the additional labels. Crowding is the inability to recognize a target object in the peripheral visual field when the target is flanked by distractors. Recognition diminishes when target-distractor distance decreases (Whitney \& Levi, 2011), which in this case happens when products are larger (Figure 4a).

Participants' eye movements were tracked using a Tobii T60 XL eye tracker with a screen resolution of 1,920 × 1,200 pixels. Average viewing distance was $60 \mathrm{~cm}$ from the screen, and a chin rest was used to stabilize head position.

\subsubsection{Data processing}

We based our re-analyses on the data processing presented in the original publication. We excluded seven participants due to insufficient data quality (calibration-related errors). The AOI margin was set to approximately $0.15^{\circ}$ and participants were classified as label or non-label users based on individual random utility models (i.e., whether they used one or both of the labels-Keyhole or organic — as criterion in the choice process). We excluded 32 participants identified as non-label users (cf. Perkovic \& Orquin, 2018). Non-label user were defined as participants whose best fitting multinomial logit model did not include information about the presence or absence of one or both labels $(\Delta$ AIC $<2)$, which does not necessarily imply a higher choice probability for products without any labels. For the remaining 39 participants, we determined target options and target attributes based on the best fitting individual choice models. We defined Keyhole label users as those for whom the choice model with the lowest AIC includes only the main effect for the presence or absence of the Keyhole label. Here, we considered each product with the Keyhole label as the target option and the Keyhole label as the target attribute. The same principle was applied to identify organic label users. Participants for whom both main effects (no interaction) are included in the choice model with the lowest AIC were identified as users of both labels. For those participants, we considered each product with both labels as the target option and the Keyhole and organic label jointly as the target attributes. Hence, the Keyhole and organic label users had four target options per choice set, whereas participants who used both labels had either one, two, or three target options depending on the co-occurrence condition. We then used target options and target attributes to determine whether participants in each trial chose a target option and fixated on the target attributes in order to build a similar data structure to Study 1 (Figure 1e). S1 were trials in which a target option was chosen, but the target attribute(s) on

\footnotetext{
${ }^{5}$ We included Study 2 in response to reviewers' comments and have preregistered the hypotheses (osf.io/8vfbe)
} 
Figure 4: Example stimuli from Perkovic and Orquin (2018) and distribution of discriminability

a
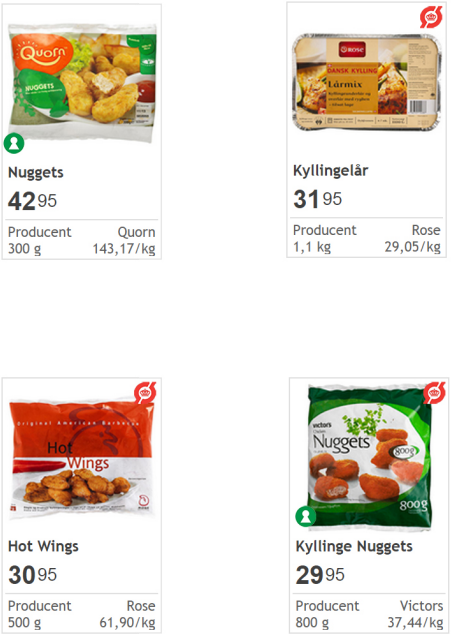

Hot Wings

3095

$\begin{array}{lr}\begin{array}{l}\text { Producent } \\ 500 g\end{array} & \text { Rose } \\ 61,90 / \mathrm{kg}\end{array}$
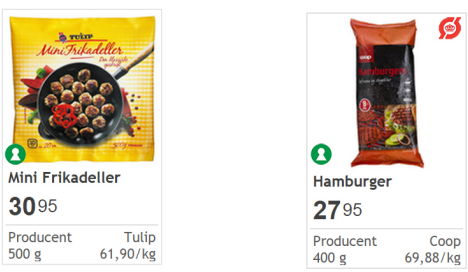
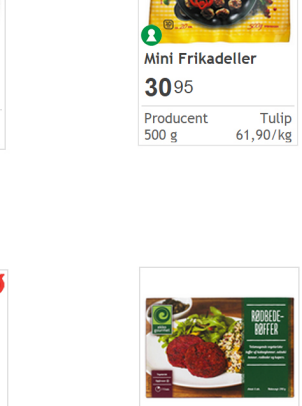

Rødbedebøffer

4295

$\begin{array}{ll}\text { Producent } \\ 340 \mathrm{~g}\end{array} \quad 126,32 / \mathrm{kg}$

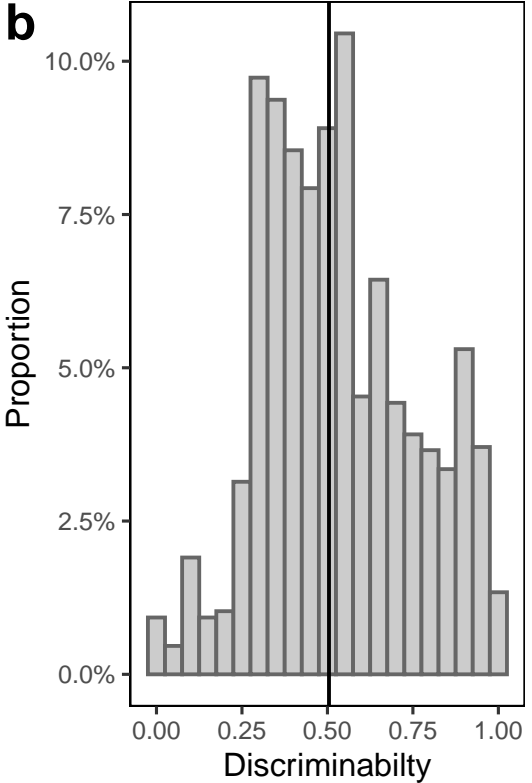

(a) Larger vs smaller products (e.g., top left vs top right) lead to varying degrees of crowding and hence target label discriminability. (b) Probability distribution of the mean discriminability of the target options across trials and participants. The vertical line represents the median $(=0.50$, mean $=0.53)$. For a colored figure, please see the online version.

the chosen target option was (were) not fixated on. S2 were trials in which no target option was chosen and no target attribute(s) on any target option was (were) fixated on. S3 were trails in which a target option was chosen and the target attribute(s) on the chosen target option was (were) fixated on. S4 were trials in which no target option was chosen but the target attribute(s) on any target option was (were) fixated on. As a measure of target-label discriminability, we used the product image size. The product image size was computed by defining a region around each product and counting the number of pixels that had an RGB value different from the background color ( $\mathrm{RGB}=[1,1,1])$. Therefore, a greater number of non-white pixels represents a larger product and, hence, a lower target attribute discriminability. To align the discriminability variable to Study 1 , we took the negative of the image size, rescaled it from 0 to 1 , and took the average across all target options in a choice set (Figure 4b).

\subsection{Confirmatory results}

Similar to Study 1, we based our analyses on the assumption that if participants were unaware of the target attribute(s), the probability of choosing a target option should approach chance level. However, due to the experimental and preferential structure of this study, chance level varies across levels of the co-occurrence condition and participants' preferences (i.e., individual choice model with lowest AIC - we identified 11 Keyhole label users, 6 organic label users, and 22 users of both labels). Therefore, we tested our preregistered hypotheses with slightly different confirmatory analyses than in Study 1: All analyses were conducted with binomial or linear mixed-effects models including fixed main effects and the interaction of the co-occurence condition (continuous: $[0,1]$ ) and participants' preferences (binary: single label vs both labels) as well as random intercepts for participant and trial. In the following, we will only specify the distribution, the criterion, and the fixed effects of interest for the respective mixed-effects models.

To quantify the role of covert attention, we fitted a binomial mixed model to participants' target choices (binary: target vs non-target) as a function of target attribute fixation (binary: fixated vs not fixated) and discriminability (continuous: $[0,1]$ ). We expected that the effect of target attribute fixation would fall into the range of the OR results in Study 1 (i.e., $1.30-3.14$ ) and interact with target label discriminability in such a way that the lower the discriminability the higher the effect of target attribute fixation. Overall, participants made $50.41 \%$ choices of the target option when the target attribute was not fixated on and almost all participants contributed to this effect (Figure 5). The model revealed a rather low and non-significant effect of target attribute fixation, $\mathrm{OR}=1.32,95 \% \mathrm{CI}=[0.56,3.12], p=0.529$, which falls close to the lower limit of the expected range. However, neither discriminability, $\mathrm{OR}=1.32,95 \% \mathrm{CI}=[0.65,2.68]$, $p=0.441$, nor the expected interaction, $\mathrm{OR}=1.35,95 \% \mathrm{CI}=[0.29,6.33], p=0.700$, had a significant effect on 
the probability of target choices. To compare the effect of discriminability between Study 1 and Study 2, we made a median split and computed ORs for the high and low discriminability conditions. In contrast to the OR results in Study 1 , the interaction of target attribute fixation with discriminability is in the opposite direction, that is, target attribute fixation had a larger effect on the probability of target choices when discriminability was high, $\mathrm{OR}=1.85$, $95 \% \mathrm{CI}=[1.12,3.06], p=0.016$, rather than low, $\mathrm{OR}=1.37,95 \% \mathrm{CI}=[0.85,2.20], p=0.196$.

To examine the effect of covert attention on participants' RTs, we fitted a linear mixed model to RTs of the trials in which participants chose a target option as a function of target attribute fixation (binary: fixated vs not fixated) and trial order (continuous: [0,49], first trial set to 0 ). We expected a large effect of covert vs overt attention with covert trials being on average shorter than overt trials, and that this reduction is larger than the time saved by not fixating on the target attribute(s). The model revealed the expected though not significant reduction in RTs for covert trials, $\mathrm{RT}=1.51$, $95 \% \mathrm{CI}=[-0.44,3.46], p=0.129$, which is similar to the reduction in Study 1 and much larger than the average dwell time on target attributes, $M_{D T}=0.24$ seconds, even when two target attributes had to be fixated as for participants who used both labels. In other words, trials where the target attribute(s) was (were) fixated on took longer than trials where the target attribute(s) was (were) not fixated on.

To examine learning effects on the use of covert attention, we fitted a binomial mixed model to participants' covert target choices (i.e., binary: S1 vs S2-S4) as a function of trial order (continuous: $[0,49]$ ). The model showed very similar results to Study 1, that is, a small increase in the probability of covert target choices for each performed trial, $\mathrm{OR}=1.01,95 \% \mathrm{CI}=[1.00,1.02], p=0.006$, starting from a relatively high prevalence of covert target choices in the first trial, $\operatorname{Pr}=0.43,95 \% \mathrm{CI}=[0.29,0.58], p=0.352$. With regard to RTs, the model terms that include trial order from the previously used linear mixed model showed a small decrease in participants' RT for each performed trial, $\mathrm{RT}=-0.10,95 \% \mathrm{CI}=[-0.13,-0.06], p<0.001$, but no significant interaction with covert attention, $\mathrm{RT}=0.03$, 95\% CI $=[-0.04,0.11], p=0.356$. Therefore, as in Study 1, the results indicate that the use of covert attention increases marginally with, but is not dependent on, task experience.

\subsection{Discussion}

In Study 2, we re-analyzed a published data set to examine whether the results of Study 1 can be replicated in a more externally valid consumer context. The results support the findings of Study 1 that participants use covert attention to include choice options based on relevant target attributes and that choices with covert attention are on average faster. However, we found no support that the use of covert attention is modulated by the discriminability of target attributes. The absence of an effect could be due to a ceiling effect. The size of the labels relative to the size of the products was about 5-10 times larger than the relative size of labels in Study 1. This could mean that even in the low discriminability condition, that is, when product images are very large, discriminability may have been higher than in the high discriminability conditions in Study 1. The larger proportion of covert choices in Study $2(50.41 \%)$ compared to Study $1(28.88 \%)$ dovetails with a ceiling effect of discriminability. The increase in covert choices compared to Study 1, could on the other hand also be due to the larger choice sets. The increase in covert choices compared to Study 1, could also be due to the larger choice sets. When choice sets become larger, consumers tend to engage in a consider-then-choose process (Payne, 1976) and covert attention might be used to efficiently form a consideration set of possible target products (Shocker et al., 1991), that is, those products that feature a relevant label, and in the following choice process, overt attention to the relevant label does not add information as only products with the label are considered.

The relatively high discriminability of the labels and specific task instructions may have enhanced the use of covert attention and, thus, limited the generalizability of the Study 2 results. The high level of discriminability presumably stems from the relative size and position of the labels to the size and position of the product images. However, it should be noted that across different markets it is common to present product labels separately and much larger in online compared to in-store shopping environments (e.g., EU organic label in Germany on rewe.de or edeka24.de, the USDA organic label in USA on walmart.com). Moreover, in-store marketing practice seems to increasingly include printing relevant labels additionally on the price tag (e.g., Føtex, Bilka, and Netto in Denmark) or signal the presence of such labels on the products with colored price tags (e.g., REWE in Germany). Therefore, the stimulus layout in Study 2 is generally representative of online and in-store environments with salient labels. However, some online stores have less salient labels (e.g. coop.dk, rema1000.dk, nemlig.com) and we surmise that the use of covert attention may be less ubiquitous in such stores.

The second factor that could have influenced the use of covert attention is the instruction to choose the healthiest option. The instruction increases the importance of the target labels and this was further compounded by the exclusion of participants who did not use the labels. This suggests that our results only generalize to consumers who use labels, but also begs the question of whether the use of covert attention was influenced by ensuring the importance of the labels. The specificity of the instruction may have encouraged participants to be more careful in their choices thereby 
increasing the use overt attention. Inspecting data from Study 1, we see that the condition where participants were instructed to choose the healthiest option (Orquin et al., 2020, Health choice) had the smallest proportion of covert choices (Table 2 and Figure 5). In sum, the high discriminability of labels and the specific task instructions may both have influenced the use of covert attention albeit in different directions. Future research should ideally disentangle these factors to provide a more precise estimate of how often an average consumer relies on covert attention.

\section{General discussion}

Decision makers are regularly faced with more choice information than they can visually attend to in a limited amount of time (Chernev et al., 2015; Perkovic \& Orquin, 2018). Many models assume that decision makers process information overtly and sequentially and therefore respond to information overload by trading off between speed and accuracy (Callaway et al., 2020; J. F. Cavanagh et al., 2014; Gluth et al., 2018; Krajbich et al., 2010). The assumption of a strictly sequential decision process operationalized through overt attention has recently been challenged by findings suggesting that covert attention can act as a precursor to overt attention (S. E. Cavanagh et al., 2019; Navalpakkam et al., 2010; Towal et al., 2013; Wästlund et al., 2018) or can even guide decision making on its own (Milosavljevic et al., 2010). To gain more insight on the issue, we examined the role of covert attention in the decision process. Across two studies containing data sets from five published articles, we extracted and evaluated the likelihood and the time of choosing a dominant option conditional on having fixated or not on the necessary attribute to identify the option as dominant.

In all data sets the dominant option(s) can only be identified visually through overtly or covertly attending to the target attribute(s) that defines (define) the option as dominant. In Study 1, we found that participants were likely to choose the dominant option even without directly gazing at the target attribute. This effect is most pronounced when the target attribute was easily detectable covertly due to high visual discriminability such as being large and salient. In one of the data sets (Orquin \& Lagerkvist, 2015, avoidance condition), the target attribute identified the non-dominant option, and here participants were likely to avoid choosing the non-dominant option without direct gazes to the target attribute. We also found that trials with accurate choices and no direct gaze were on average 1.50 seconds faster than trials with accurate choices and direct gaze at the target attribute. Some of this difference could be due to the time saved by not gazing at the target attribute. However, the response time difference of 1.50 seconds is larger than the average time participants spent fixating on the target attribute, 0.55 seconds, which leaves an explanatory gap of 0.95 seconds. In Study 2, we demonstrated that the findings of Study 1 generalize to more naturalistic decision situations with larger choice sets (but please consider potential limitations as discussed in Study 2). We found that participants were even more likely to choose the dominant option without directly gazing at the target attribute and that they again saved about 1.50 seconds compared to overt choices. We believe that the response time reduction is a consequence of using covert attention which can act as distributed attention. Distributed attention allows attending to and processing multiple attributes in parallel and we know from previous research that in situations that facilitate parallel processing of information, for instance, to limit search to a subset of candidate target options (Wolfe et al., 1989) or in cases in which target options are defined by a single feature (Treisman \& Gelade, 1980), distributed attention increases speed in visual-search tasks. Our findings suggest that decision makers are likely using the same process, namely covert distributed attention, to make faster choices that are well above chance levels in terms of accuracy.

\subsection{Theoretical implications}

Our findings show that covert distributed attention can play an important role in the decision-making process beyond that of being a precursor of subsequent processes such as overt attention. Formulated in terms of contemporary gazeinformed sequential sampling models, it might be possible that the neural value signal (Hare et al., 2011; McGinty et al., 2016) of a not-gazed-at option does not diminish or even increases while another option is overtly attended to (cf. Ashby et al., 2016). This interpretation is corroborated by recent results by Smith and Krajbich (2018) who demonstrated that the relation between gaze, choice, and response time correlates with measures of the breadth of participants' attentional scope. According to those results, the relation between gaze and choice decreases with increasing breadth of attentional scope. Combining those results, future gaze-informed sequential sampling models of preferential choice could incorporate covert attention, for instance, as an additional parameter for the not-gazed at option based on distance, salience, and individual attentional scope. A related approach has already been taken by Towal et al. (2013) who used salience and preference information to predict sequences of overt attention operationalized as gazes. We know that factors such as salience guide overt attention (for a review, see Wolfe \& Horowitz, 2017), and the work of Towal et al. (2013) accounts for covert attention as a precursor to overt attention. We argue, however, that this does not seem to be sufficient given our findings. Covert distributed attention seems to play a role on its own apart from being a precursor for overt attention and it is an important question to determine the boundary conditions of switching between the two. For instance, when does highly salient information in the decision environment attract covert instead of overt attention? Given prior literature, we would expect that learning is an important moderator that is, familiarity or experience with 
the available information in the decision environment. Since our findings do not strongly support learning effects, future research should develop paradigms to directly test if and how learning plays a role in covert attention and experimentally examine the conditions that facilitate or impede learning of covert attention in preferential decision making.

Our findings also suggest a novel candidate mechanism driving intuitive (automatic) in contrast to conscious (deliberate) decision processes (Melnikoff \& Bargh, 2018). We know that automatic processes are associated with fast, yet accurate decisions (Glöckner \& Herbold, 2011; Horstmann et al., 2009), and that such processes can be hindered by the research method. Glöckner and Betsch (2008b) showed that decision makers are able to integrate a vast amount of information extremely quickly when information search is not restricted by the experimental procedure, such as open vs closed information board paradigms. Based on this finding, the authors argued that most of the evidence for deliberate processes is an artefact of the method; however, when information search is unconstrained, decision makers rely on automatic processes integrating all available information in parallel (Glöckner \& Betsch, 2008a, 2008b). Thus, combined with our findings, it seems plausible that automatic decision making crucially depends on covert distributed attention playing a role on its own apart from being a precursor to overt attention.

\subsection{Methodological implications}

Our findings challenge current research practices regarding the use of eye tracking to understand psychological processes. It is commonly assumed that direct gaze to a stimulus implies that the stimulus has been cognitively processed whereas the absence of direct gaze implies the opposite (Orquin \& Holmqvist, 2018). This assumption is the foundation of process-tracing research aiming to identify decision processes through process measures such as overt attention (Schoemann et al., 2019; Schulte-Mecklenbeck et al., 2017). Our findings suggest that researchers must exercise caution before using the absence of eye movements to a stimulus to conclude that the stimulus has not been visually identified or cognitively processed. This important point is of particular importance for all gaze-informed decision models not controlling for covert attention and has recently been raised (Mormann \& Russo, 2021; Schonberg \& Katz, 2020). ${ }^{6}$ One way to address the question is with gaze-contingent paradigms which allows the researcher to restrict the visual discriminability around the point of gaze (Franco-Watkins \& Johnson, 2011a, 2011b). By in- or decreasing the discriminability of the peripheral visual field, this approach can restrict the use of covert attention and examine the relation between overt and covert attention. Another challenge is that process models often assume that information is processed sequentially which makes response time measures useful for investigating decision strategies associated with speed-accuracy trade-offs (Payne et al., 1988; Ratcliff, 1978). However, our findings question the assumption of sequential information processing and show a need for further research to better understand the extent of parallel processing. Since our findings are based on aggregate-level analysis, future research should develop paradigms to disentangle accurate choices resulting from covert attention vs chance at the level of individual trials. This would be particularly relevant from a process-tracing perspective as such paradigms could explain the reduction in response times from the use of covert vs overt attention and identify associated gaze behavior that goes beyond missing direct fixations to relevant information. It is, for instance, plausible that covert choices are associated with a more global information processing style, that is, longer saccades and possibly shorter fixations while overt choices are associated with more local processing (Liechty et al., 2003).

\subsection{Practical implications}

We have shown here that harnessing covert attention by increasing the visual discriminability of important product information can help decision makers to save time and still make accurate decisions. Health, sustainability, or safety symbols are ideal candidates for covert attention because it is sufficient to visually identify their presence to infer their meaning. Many other types of information, such as complex textual descriptions, may not lend themselves to covert distributed attention and thus require direct overt attention. By bridging insights on the distribution of overt attention with insights about what can and cannot be identified covertly, product designers can optimize designs for information uptake.

It is, for instance, inefficient to place features that can be identified covertly in locations that are likely to attract overt attention. Brands and logos are examples of product features that may be identified covertly, yet they are typically placed squarely in the middle of products where decision makers are most likely to attend overtly (Orquin et al., 2020).

\footnotetext{
6“"The [attentional drift diffusion model (aDDM)] has influenced a number of groups [35-37], but it should be noted that the locus of attention in the model is determined by overt attention (i.e., gaze position) and not by controlling for covert attention, which might sometimes be deployed elsewhere [38]. Whether or not manipulations of covert attention influence preference similarly to manipulations of overt attention, is an important open question." (Schonberg and Katz, 2020, p. 506)

"The aDDM assumes that attention is identical to looking. However, and without getting into the current debates on attention itself (e.g., [23,66]), what is looked at, the object of regard, may not be what is being thought about, the object of internal processing ([67])." (Mormann and Russo, 2021, p. 308)
} 
Consumers spend around 0.55 seconds attending to a product in the supermarket (Gidlöf et al., 2013) and we have shown here, albeit in laboratory studies, that overtly attending to a target attribute can take as much as 0.54 seconds. There is, therefore, much to be gained in terms of efficiency by increasing information discriminability thus allowing consumers to covertly attend to product information. Future research should examine the possibilities and limitations of covert attention for enhancing efficient consumer information processing.

Our findings are also relevant to the design of advertising. Previous research has focused entirely on how to attract overt attention to the ad and particularly the brand (Orquin \& Wedel, 2020; Pieters et al., 2007), but we believe that covert attention may play a similar important role in advertising. It is, for instance, possible that consumers attend covertly to ad elements like the brand in much the same way they attend covertly to product elements like labels. One clue that covert attention plays a role in advertising is that ads with high visual discriminability are more likely to attract overt attention (Pieters et al., 2007). This suggests that ads with high discriminability are more likely to be detected in the peripheral visual field using covert attention. Covert attention is also a likely candidate for explaining banner blindness, that is, the ability of decision makers to avoid directing overt attention to banner ads (Chiu et al., 2017). This suggests that advertisers may need to be cautious when designing ads that can be detected covertly. A better understanding of the relation between overt and covert attention is therefore useful for optimizing the design of ads and we propose that future research on advertising effectiveness should move away from a narrow focus on overt attention to a broader view of information uptake through both overt and covert attention.

\subsection{Conclusion}

We have demonstrated that decision makers can make fast and accurate decisions even when not gazing directly at the relevant information. We argue that covert attention has a function in itself, and that overt gaze behavior is not a sufficient indicator of information processing. Our findings show new avenues for improving consumer information processing, but also challenge recent gaze-informed sequential sampling models and call for further research enhancing our understanding of the role of covert attention in decision making.

\section{References}

Armel, K. C., Beaumel, A., \& Rangel, A. (2008). Biasing simple choices by manipulating relative visual attention. Judgment and Decision making, 3(5), 396-403.

Ashby, N. J., Jekel, M., Dickert, S., \& Glöckner, A. (2016). Finding the right fit: A comparison of process assumptions underlying popular drift-diffusion models. Journal of Experimental Psychology: Learning, Memory, and Cognition, 42(12), 1982. https://doi.org/10.1037/xlm0000279

Bartels, M., Tillack, K., \& Lin, C.-T. J. (2018). Communicating nutrition information at the point of purchase: An eye-tracking study of shoppers at two grocery stores in the united states. International Journal of Consumer Studies, 42(5), 557-565. https://doi.org/10.1111/ijcs.12474

Borenstein, M., Hedges, L. V., Higgins, J. P., \& Rothstein, H. R. (2011). Introduction to meta-analysis. John Wiley \& Sons. https://doi.org/10.1002/9780470743386

Busemeyer, J. R., Gluth, S., Rieskamp, J., \& Turner, B. M. (2019). Cognitive and neural bases of multi-attribute, multi-alternative, value-based decisions. Trends in Cognitive Sciences, 23(3), 251-263. https://doi.org/10. 1016/j.tics.2018.12.003

Callaway, F., Rangel, A., \& Griffiths, T. (2020). Attention in value-based choice as optimal sequential sampling. https://doi.org/10.31234/osf.io/57v6k

Carrasco, M. (2011). Visual attention: The past 25 years. Vision research, 51(13), 1484-1525. https://doi.org/10.1016/j. visres.2011.04.012

Carter, E. C., Schönbrodt, F. D., Gervais, W. M., \& Hilgard, J. (2019). Correcting for bias in psychology: A comparison of meta-analytic methods. Advances in Methods and Practices in Psychological Science, 2(2), 115-144. https://doi.org/10.1177/2515245919847196

Cavanagh, J. F., Wiecki, T. V., Kochar, A., \& Frank, M. J. (2014). Eye tracking and pupillometry are indicators of dissociable latent decision processes. Journal of Experimental Psychology: General, 143(4), 1476-1488. https://doi.org/10.1037/a0035813

Cavanagh, S. E., Malalasekera, W. N., Miranda, B., Hunt, L. T., \& Kennerley, S. W. (2019). Visual fixation patterns during economic choice reflect covert valuation processes that emerge with learning. Proceedings of the National Academy of Sciences, 116(45), 22795-22801. https://doi.org/10.1073/pnas.1906662116

Chernev, A., Böckenholt, U., \& Goodman, J. (2015). Choice overload: A conceptual review and meta-analysis. Journal of Consumer Psychology, 25(2), 333-358. https://doi.org/10.1016/j.jcps.2014.08.002 
Chiu, Y.-P., Lo, S.-K., \& Hsieh, A.-Y. (2017). How colour similarity can make banner advertising effective: Insights from gestalt theory. Behaviour \& Information Technology, 36(6), 606-619. https://doi.org/10.1080/0144929X. 2016.1267264

Colas, J. T. (2017). Value-based decision making via sequential sampling with hierarchical competition and attentional modulation. PloS ONE, 12(10), e0186822. https://doi.org/10.1371/journal.pone.0203093

Dosso, J. A., Huynh, M., \& Kingstone, A. (2020). I spy without my eye: Covert attention in human social interactions. Cognition, 202, 104388. https://doi.org/10.1016/j.cognition.2020.104388

Dutilh, G., \& Rieskamp, J. (2016). Comparing perceptual and preferential decision making. Psychonomic Bulletin \& Review, 23(3), 723-737. https://doi.org/10.3758/s13423-015-0941-1

Evans, K. K., Georgian-Smith, D., Tambouret, R., Birdwell, R. L., \& Wolfe, J. M. (2013). The gist of the abnormal: Above-chance medical decision making in the blink of an eye. Psychonomic Bulletin \& Review, 20(6), 11701175. https://doi.org/10.3758/s13423-013-0459-3

Fisher, G. (2017). An attentional drift diffusion model over binary-attribute choice. Cognition, 168, 34-45. https: //doi.org/10.1016/j.cognition.2017.06.007

Fisher, G. (2021). Intertemporal choices are causally influenced by fluctuations in visual attention. Management Science, 67(8), 4961-4981. https://doi.org/10.1287/mnsc.2020.3732

Forstmann, B. U., Ratcliff, R., \& Wagenmakers, E. (2016). Sequential sampling models in cognitive neuroscience: Advantages, applications, and extensions. Annual Review of Psychology, 67, 641-666. https://doi.org/10.1146/ annurev-psych-122414-033645

Franco-Watkins, A. M., \& Johnson, J. G. (2011a). Applying thedecision moving windowto risky choice: Comparisonof eye-tracking and mouse-tracing methods. Judgment and Decision Making, 6(8), 740-749.

Franco-Watkins, A. M., \& Johnson, J. G. (2011b). Decision moving window: Using interactive eye tracking to examine decision processes. Behavior Research Methods, 43(3), 853. https://doi.org/10.3758/s13428-011-0083-y

Ghaffari, M., \& Fiedler, S. (2018). The power of attention: Using eye gaze to predict other-regarding and moral choices. Psychological Science, 29(11), 1878-1889. https://doi.org/10.1177/0956797618799301

Gidlöf, K., Wallin, A., Dewhurst, R., \& Holmqvist, K. (2013). Using eye tracking to trace a cognitive process: Gaze behaviour during decision making in a natural environment. Journal of Eye Movement Research, 6(1), 1-14. https://doi.org/10.16910/jemr.6.1.3

Glöckner, A., \& Herbold, A. (2011). An eye-tracking study on information processing in risky decisions: Evidence for compensatory strategies based on automatic processes. Journal of Behavioral Decision Making, 24(1), 71-98. https://doi.org/10.1002/bdm.684

Glöckner, A., \& Betsch, T. (2008a). Do people make decisions under risk based on ignorance? an empirical test of the priority heuristic against cumulative prospect theory. Organizational Behavior and Human Decision Processes, 107(1), 75-95. https://doi.org/10.1016/j.obhdp.2008.02.003

Glöckner, A., \& Betsch, T. (2008b). Multiple-reason decision making based on automatic processing. Journal of experimental psychology: Learning, memory, and cognition, 34(5), 1055-1075. https://doi.org/10.1037/02787393.34.5.1055

Gluth, S., Kern, N., Kortmann, M., \& Vitali, C. L. (2020). Value-based attention but not divisive normalization influences decisions with multiple alternatives. Nature Human Behaviour, 4(6), 634-645. https://doi.org/10.1038/s41562020-0822-0

Gluth, S., Spektor, M. S., \& Rieskamp, J. (2018). Value-based attentional capture affects multi-alternative decision making. Elife, 7, e39659. https://doi.org/10.7554/eLife.39659.001

Greene, M. R., \& Oliva, A. (2009). The briefest of glances: The time course of natural scene understanding. Psychological Science, 20(4), 464-472. https://doi.org/10.1111/j.1467-9280.2009.02316.x

Hare, T. A., Malmaud, J., \& Rangel, A. (2011). Focusing attention on the health aspects of foods changes value signals in vmpfc and improves dietary choice. Journal of neuroscience, 31(30), 11077-11087. https://doi.org/10.1523/ JNEUROSCI.6383-10.2011

Hausegger, T., Vater, C., \& Hossner, E.-J. (2019). Peripheral vision in martial arts experts: The cost-dependent anchoring of gaze. Journal of Sport and Exercise Psychology, 41(3), 137-145. https://doi.org/10.1123/jsep.2018-0091

Horstmann, N., Ahlgrimm, A., \& Glöckner, A. (2009). How distinct are intuition and deliberation? an eye-tracking analysis of instruction-induced decision modes. Judgment and Decision Making, 4(5), 335-354.

Jekel, M., Glöckner, A., \& Bröder, A. (2018). A new and unique prediction for cue-search in a parallel-constraint satisfaction network model: The attraction search effect. Psychological review, 125(5), 744. https://doi.org/10. $1037 /$ rev0000107

Kato, T. (2020). Using "enzan no metsuke" (gazing at the far mountain) as a visual search strategy in kendo. Frontiers in Sports and Active Living, 2, 40. https://doi.org/10.3389/fspor.2020.00040

Krajbich, I., Armel, C., \& Rangel, A. (2010). Visual fixations and the computation and comparison of value in simple choice. Nature Neuroscience, 13(10), 1292-1298. https://doi.org/10.1038/nn.2635 
Krajbich, I. (2019). Accounting for attention in sequential sampling models of decision making. Current opinion in psychology, 29, 6-11. https://doi.org/10.1016/j.copsyc.2018.10.008

Krajbich, I., \& Rangel, A. (2011). Multialternative drift-diffusion model predicts the relationship between visual fixations and choice in value-based decisions. Proceedings of the National Academy of Sciences, 108(33), 13852-13857. https://doi.org/10.1073/pnas.1101328108

Kunar, M. A., Watson, D. G., Tsetsos, K., \& Chater, N. (2017). The influence of attention on value integration. Attention, Perception, \& Psychophysics, 79(6), 1615-1627. https://doi.org/10.3758/s13414-017-1340-7

Liechty, J., Pieters, R., \& Wedel, M. (2003). Global and local covert visual attention: Evidence from a bayesian hidden markov model. Psychometrika, 68(4), 519-541. https://doi.org/10.1007/bf02295608

Lim, S.-L., O'Doherty, J. P., \& Rangel, A. (2011). The decision value computations in the vmPFC and striatum use a relative value code that is guided by visual attention. Journal of Neuroscience, 31(37), 13214-13223. https://doi.org/10.1523/jneurosci.1246-11.2011

Liu, H.-Z., Zhou, Y.-B., Wei, Z.-H., \& Jiang, C.-M. (2020). The power of last fixation: Biasing simple choices by gaze-contingent manipulation. Acta Psychologica, 208(February), 103106. https://doi.org/10.1016/j.actpsy. 2020.103106

Martin, J., Nenycz-Thiel, M., Dawes, J., Tanusondjaja, A., Cohen, J., McColl, B., \& Trinh, G. (2020). Fundamental basket size patterns and their relation to retailer performance. Journal of Retailing and Consumer Services, 54, 102032. https://doi.org/10.1016/j.jretconser.2020.102032

McGinty, V. B., Rangel, A., \& Newsome, W. T. (2016). Orbitofrontal Cortex Value Signals Depend on Fixation Location during Free Viewing. Neuron, 90(6), 1299-1311. https://doi.org/10.1016/j.neuron.2016.04.045

Melnikoff, D. E., \& Bargh, J. A. (2018). The mythical number two. Trends in cognitive sciences, 22(4), $280-293$. https://doi.org/10.1016/j.tics.2018.02.001

Milosavljevic, M., Malmaud, J., Huth, A., Koch, C., \& Rangel, A. (2010). The drift diffusion model can account for value-based choice response times under high and low time pressure. Judgment and Decision Making, 5(6), 437-449.

Milosavljevic, M., Navalpakkam, V., Koch, C., \& Rangel, A. (2012). Relative visual saliency differences induce sizable bias in consumer choice. Journal of Consumer Psychology, 22(1), 67-74. https://doi.org/10.1016/j.jcps.2011. 10.002

Mormann, M., \& Russo, J. E. (2021). Does Attention Increase the Value of Choice Alternatives? Trends in Cognitive Sciences, 25(4), 305-315. https://doi.org/10.1016/j.tics.2021.01.004

Navalpakkam, V., Koch, C., Rangel, A., \& Perona, P. (2010). Optimal reward harvesting in complex perceptual environments. Proceedings of the National Academy of Sciences, 107(11), 5232-5237. https://doi.org/10.1073/ pnas.0911972107

Oliva, A. (2005). Gist of the scene. Neurobiology of attention (pp. 251-256). Elsevier. https://doi.org/10.1016/B978012375731-9/50045-8

Orquin, J. L., Ashby, N., \& Clarke, A. (2016). Areas of interest as a signal detection problem in behavioral eye-tracking research. Journal of Behavioral Decision Making, 29, 103-115. https://doi.org/10.1002/bdm.1867

Orquin, J. L., Bagger, M. P., Lahm, E. S., Grunert, K. G., \& Scholderer, J. (2020). The visual ecology of product packaging and its effects on consumer attention. Journal of Business Research, 111, 187-195. https://doi.org/ 10.1016/j.jbusres.2019.01.043

Orquin, J. L., Bagger, M. P., \& Mueller Loose, S. (2013). Learning affects top down and bottom up modulation of eye movements in decision making. Judgment and Decision making, 8(6), 700-716.

Orquin, J. L., \& Holmqvist, K. (2018). Threats to the validity of eye-movement research in psychology. Behavior Research Methods, 50(4), 1645-1656. https://doi.org/10.3758/s13428-017-0998-z

Orquin, J. L., \& Lagerkvist, C. J. (2015). Effects of salience are both short- and long-lived. Acta Psychologica, 160, 69-76. https://doi.org/10.1016/j.actpsy.2015.07.001

Orquin, J. L., Lahm, E. S., \& Stojic, H. (2021). The visual environment and attention in decision making. Psychological Bulletin, 147(6), 597-617. https://doi.org/10.1037/bul0000328

Orquin, J. L., \& Wedel, M. (2020). Contributions to attention based marketing: Foundations, insights, and challenges. Journal of Business Research, 111, 85-90. https://doi.org/10.1016/j.jbusres.2020.02.012

Pärnamets, P., Johansson, P., Hall, L., Balkenius, C., Spivey, M., \& Richardson, D. (2015). Biasing moral decisions by exploiting the dynamics of eye gaze. Proceedings of the National Academy of Sciences, 112(13), 4170-4175. https://doi.org/10.1073/pnas.1415250112

Payne, J. W. (1976). Task complexity and contingent processing in decision making: An information search and protocol analysis. Organizational Behavior and Human Performance, 16(2), 366-387. https://doi.org/10.1016/00305073(76)90022-2

Payne, J. W., Bettman, J. R., \& Johnson, E. J. (1988). Adaptive strategy selection in decision making. Journal of experimental psychology: Learning, Memory, and Cognition, 14(3), 534-552. https://doi.org/10.1037/02787393.14.3.534 
Perkovic, S., \& Orquin, J. L. (2018). Implicit statistical learning in real-world environments leads to ecologically rational decision making. Psychological Science, 29(1), 34-44. https://doi.org/10.1177/0956797617733831

Peschel, A. O., Orquin, J. L., \& Loose, S. M. (2019). Increasing consumers' attention capture and food choice through bottom-up effects. Appetite, 132, 1-7. https://doi.org/10.1016/j.appet.2018.09.015

Pieters, R., Wedel, M., \& Zhang, J. (2007). Optimal feature advertising design under competitive clutter. Management Science, 53(11), 1815-1828. https://doi.org/10.1287/mnsc.1070.0732

Posner, M. I. (1980). Orienting of attention. Quarterly journal of experimental psychology, 32(1), 3-25. https://doi.org/ $10.1080 / 00335558008248231$

R Core Team. (2020). R: A language and environment for statistical computing. R Foundation for Statistical Computing. Vienna, Austria. https://www.r-project.org/

Ratcliff, R. (1978). A theory of memory retrieval. Psychological review, 85(2), 59-108. https://doi.org/10.1037/0033295X.85.2.59

Ratcliff, R., Smith, P. L., Brown, S. D., \& McKoon, G. (2016). Diffusion decision model: Current issues and history. Trends in cognitive sciences, 20(4), 260-281. https://doi.org/10.1016/j.tics.2016.01.007

Rovamo, J., \& Raninen, A. (1984). Critical flicker frequency and m-scaling of stimulus size and retinal illuminance. Vision research, 24(10), 1127-1131. https://doi.org/10.1016/0042-6989(84)90166-4

Schoemann, M., Schulte-Mecklenbeck, M., Renkewitz, F., \& Scherbaum, S. (2019). Forward inference in risky choice: Mapping gaze and decision processes. Journal of Behavioral Decision Making, 32(5), 521-535. https://doi.org/10.1002/bdm.2129

Schonberg, T., \& Katz, L. N. (2020). A neural pathway for nonreinforced preference change. Trends in Cognitive Sciences, 24(7), 504-514. https://doi.org/10.1016/j.tics.2020.04.002

Schulte-Mecklenbeck, M., Kühberger, A., Gagl, B., \& Hutzler, F. (2017). Inducing thought processes: Bringing process measures and cognitive processes closer together. Journal of Behavioral Decision Making, 30(5), 1001-1013. https://doi.org/10.1002/bdm.2007

Shimojo, S., Simion, C., Shimojo, E., \& Scheier, C. (2003). Gaze bias both reflects and influences preference. Nature Neuroscience, 6(12), 1317-1322. https://doi.org/10.1038/nn1150

Shocker, A. D., Ben-Akiva, M., Boccara, B., \& Nedungadi, P. (1991). Consideration set influences on consumer decision-making and choice: Issues, models, and suggestions. Marketing Letters, 2(3), 181-197. https: //doi.org/10.1007/BF00554125

Smith, S. M., \& Krajbich, I. (2018). Attention and choice across domains. Journal of Experimental Psychology: General, 147(12), 1810-1826. https://doi.org/10.1037/xge0000482

Smith, S. M., \& Krajbich, I. (2019). Gaze Amplifies Value in Decision Making. Psychological Science, 30(1), 116-128. https://doi.org/10.1177/0956797618810521

Stüttgen, P., Boatwright, P., \& Monroe, R. T. (2012). A satisficing choice model. Marketing Science, 31(6), 878-899. https://doi.org/10.1287/mksc.1120.0732

Tavares, G., Perona, P., \& Rangel, A. (2017). The attentional drift diffusion model of simple perceptual decision-making. Frontiers in Neuroscience, 11. https://doi.org/10.3389/fnins.2017.00468

Thomas, A. W., Molter, F., \& Krajbich, I. (2021). Uncovering the computational mechanisms underlying manyalternative choice. eLife, 10. https://doi.org/10.7554/elife.57012

Thomas, A. W., Molter, F., Krajbich, I., Heekeren, H. R., \& Mohr, P. N. C. (2019). Gaze bias differences capture individual choice behaviour. Nature Human Behaviour, 3(6), 625-635. https://doi.org/10.1038/s41562-0190584-8

Towal, R. B., Mormann, M., \& Koch, C. (2013). Simultaneous modeling of visual saliency and value computation improves predictions of economic choice. Proceedings of the National Academy of Sciences, 110(40), E3858E3867. https://doi.org/10.1073/pnas.1304429110

Treisman, A. M., \& Gelade, G. (1980). A feature-integration theory of attention. Cognitive psychology, 12(1), 97-136. https://doi.org/10.1016/0010-0285(80)90005-5

Turner, B. M., Schley, D. R., Muller, C., \& Tsetsos, K. (2018). Competing theories of multialternative, multiattribute preferential choice. Psychological Review, 125(3), 329-362. https://doi.org/10.1037/rev0000089

Wästlund, E., Shams, P., \& Otterbring, T. (2018). Unsold is unseen... or is it? examining the role of peripheral vision in the consumer choice process using eye-tracking methodology. Appetite, 120, 49-56. https://doi.org/10.1016/j. appet.2017.08.024

Whitney, D., \& Levi, D. M. (2011). Visual crowding: A fundamental limit on conscious perception and object recognition. Trends in Cognitive Sciences, 15(4), 160-168. https://doi.org/10.1016/j.tics.2011.02.005

Wolfe, J. M. (1994). Guided search 2.0 a revised model of visual search. Psychonomic Bulletin \& Review, 1(2), 202-238. https://doi.org/10.3758/BF03200774

Wolfe, J. M., Cave, K. R., \& Franzel, S. L. (1989). Guided search: An alternative to the feature integration model for visual search. Journal of Experimental Psychology: Human perception and performance, 15(3), 419-433. https://doi.org/10.1037/0096-1523.15.3.419 
Wolfe, J. M., \& Horowitz, T. S. (2017). Five factors that guide attention in visual search. Nature Human Behaviour, 1(3). https://doi.org/10.1038/s41562-017-0058

\section{A Detailed description of data sets in Study 1}

In all data sets, participants made a series of one-shot choices between two to four choice options. In three out of four data sets (Orquin et al., 2020; Orquin \& Lagerkvist, 2015; Peschel et al., 2019), one option is always considered the target option which means that it dominates the non-target option on the account of a single target attribute. For instance, in Peschel et al. (2019) participants made a series of choices between different pairs of food products and a no-choice option. Each pair was composed to reduce differences in subjective preferences between options so that on average participants were indifferent between the options. In critical trials (i.e., the trials including an experimental manipulation), the target option featured the organic label (i.e., the target attribute). The target option therefore dominated the non-target option on account of the target attribute (see Figure 1). The appearance of the target attribute was manipulated across trials being either small and of low salience or large and of high salience, but manipulations differed between data sets (see https://osf.io/nfudq for detailed illustrations of the different appearances of the stimuli for each data set). The difference in size and salience (i.e., discriminability) means that the large and more salient attributes were easier to detect in the peripheral visual field than the smaller and less salient ones (Rovamo \& Raninen, 1984). In two data sets (Orquin et al., 2020; Orquin \& Lagerkvist, 2015), the subjective value of the target attribute varied depending on a top-down between-subjects condition. In Orquin and Lagerkvist (2015), participants were instructed about the meaning of the target attribute. Participants were informed that the label indicated a sustainable product (approach condition), indicated a genetically modified food product (avoidance condition), or received no information about the label (control condition). We did not include the control condition in the re-analysis since in this condition the label does not discriminate between choice options. In Study 2, participants were instructed to either make a choice according to their own preferences (control choice), choose the healthiest product (health choice), or were primed with health goals and then instructed to choose according to their own preferences (primed choice). The target attributes were health-related labels. In Orquin et al. (2013), there was no designated target attribute or target option and a different discriminability manipulation. Participants made choices between four options that differed on six attributes and a no-choice option. To identify a target option, we first fitted a random utility model for each participant. From this, we computed the individual-specific utilities for each option in a given trial. The option with the highest utility was considered the target option; participants chose it with a very high probability (mean target choice $=.91$ ). To identify a target attribute, we computed the attribute importance (the proportional weight of each attribute) from the random utility model. The attribute with the largest importance was considered the target attribute (mean attribute importance $=48.64 \%$ ). Regarding discriminability, the visual information matrix condition constitutes the low discriminability condition and the product representation condition constitutes the high discriminability condition. An overview of all datasets is shown in Table 1. 


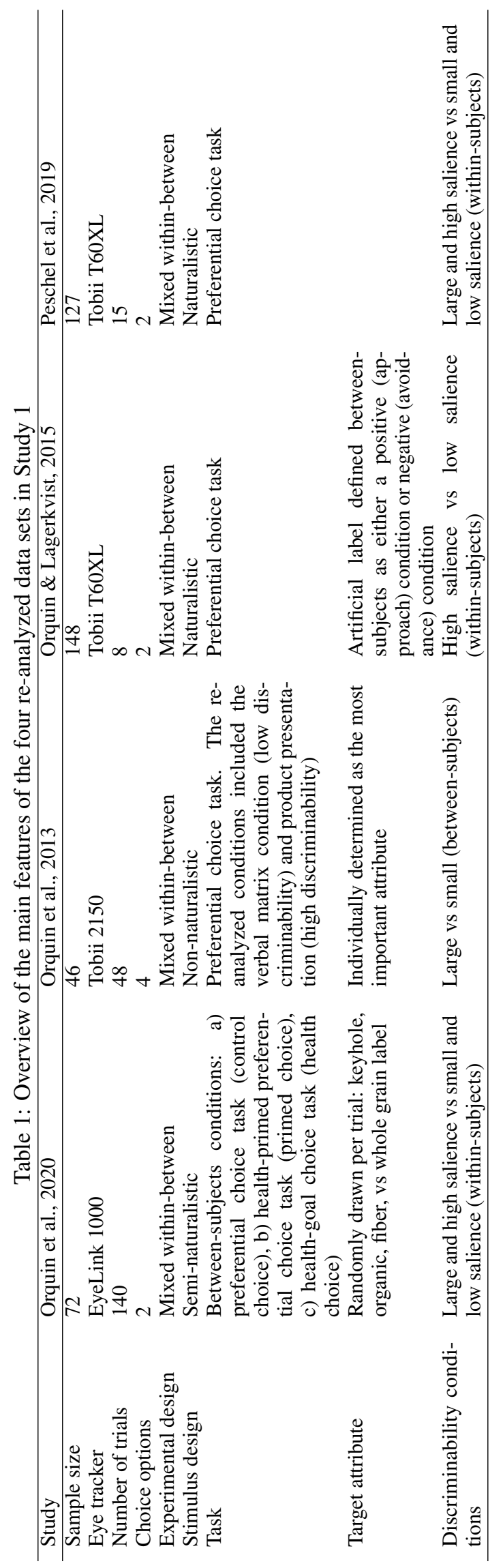




\section{B Additional statistics}

Figure 5: Percentage of covert trials (see Figure 1e, S1) on the participant level for all five re-analyzed data sets
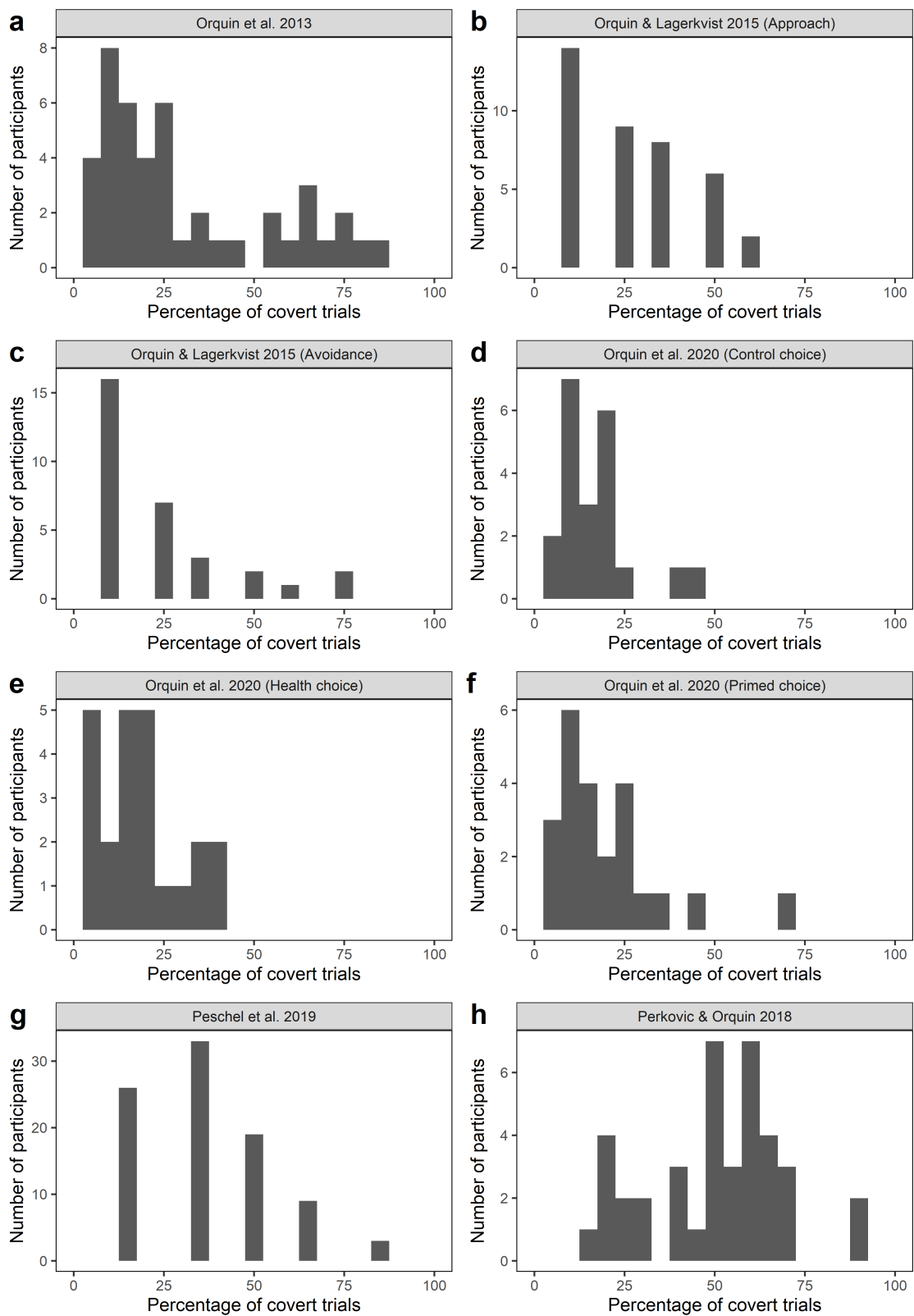
Table 2: Results of modelling participants' covert target choices as a function of trial order

\begin{tabular}{lrrrrrr}
\hline & \multicolumn{3}{c}{ Intercept } & \multicolumn{3}{c}{ Trial order } \\
Data set & $b$ & $\mathrm{SE}$ & $p$ & $b$ & $\mathrm{SE}$ & $p$ \\
\hline Orquin et al. 2020 (Health) & -2.16 & 0.25 & $<.001$ & 0.01 & 0.00 & $<.001$ \\
Orquin et al. 2020 (Control) & -1.44 & 0.25 & $<.001$ & 0.00 & 0.00 & 0.216 \\
Orquin et al. 2020 (Primed) & -1.67 & 0.26 & $<.001$ & 0.01 & 0.00 & 0.002 \\
Orquin \& Lagerkvist 2015 (Ap.) & 0.27 & 0.38 & 0.474 & -1.49 & 0.55 & 0.007 \\
Orquin \& Lagerkvist 2015 (Av.) & -1.00 & 0.47 & 0.033 & -0.95 & 0.64 & 0.139 \\
Orquin et al. 2013 & -0.14 & 0.18 & 0.422 & -0.27 & 0.20 & 0.188 \\
Peschel et al. 2019 & 0.12 & 0.23 & 0.595 & -0.14 & 0.36 & 0.693 \\
Perkovic \& Orquin 2018 & -0.30 & 0.32 & 0.352 & 0.01 & 0.00 & 0.006 \\
\hline
\end{tabular}

Note: For the Perkovic \& Orquin (2018) data set we used a different model structure, please see the main text for details. 


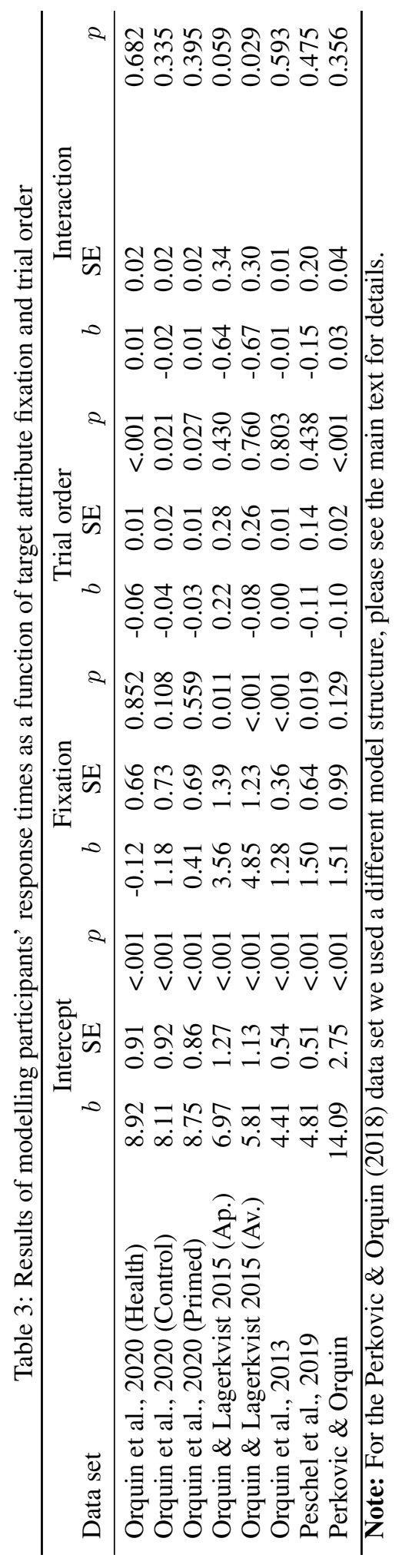




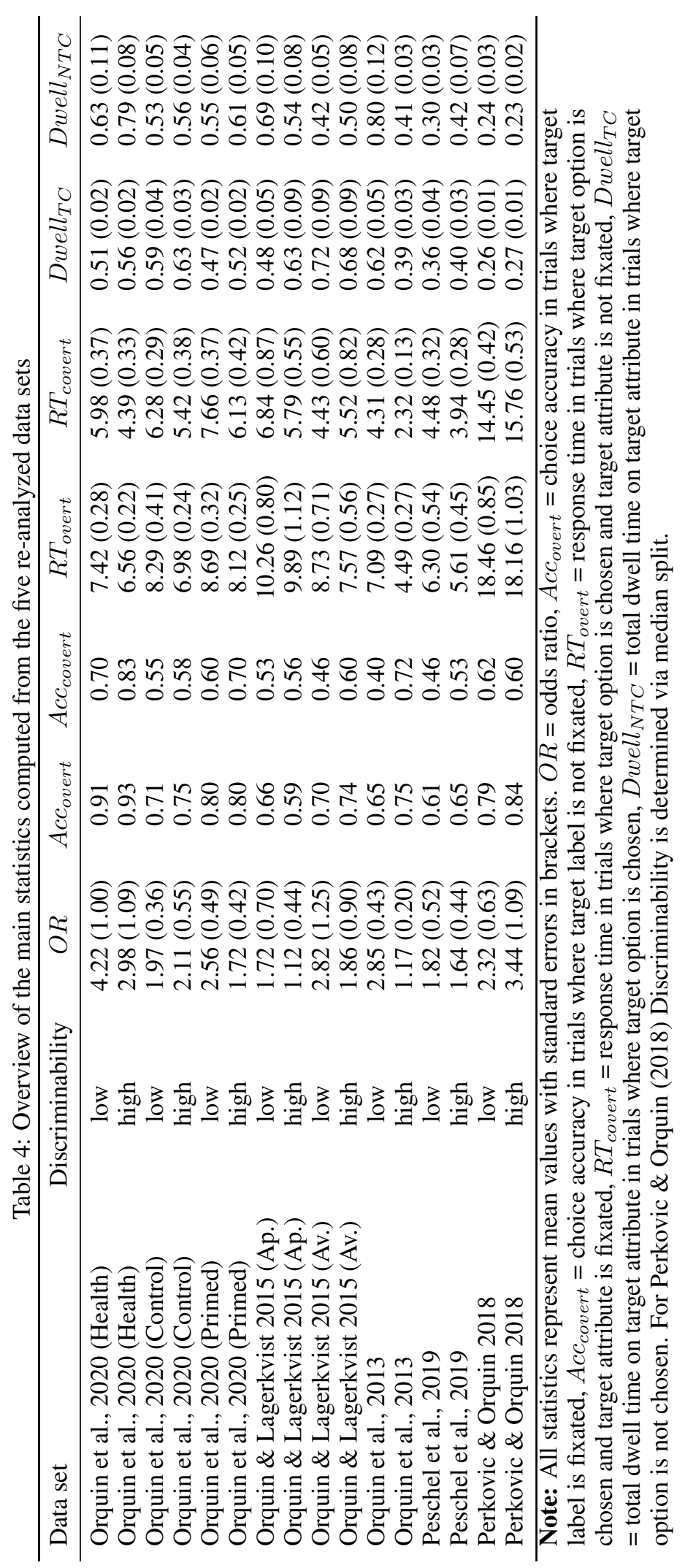

